\title{
Short communication: Selection for persistency by changing the shape of lactation curves with geometric morphometrics
}

\author{
Angel-Amed Durón-Benítez, ${ }^{1 *}$ Joel Ira Weller, ${ }^{1} \dagger$ and Ephraim Ezra ${ }^{2}$ \\ ${ }^{1}$ Institute of Animal Sciences, Agricultural Research Organization, The Volcani Center, Rishon LeZion 7505101, Israel \\ ${ }^{2}$ Israeli Cattle Breeders Association, Caesarea Industrial Park 3088900, Israel
}

\section{ABSTRACT}

The objective was to simulate the response to selection by modifying the shape of the lactation curve to improve persistency in Israeli first-parity Holstein cattle using geometric morphometrics and multivariate quantitative genetics. The 2-dimensional Cartesian coordinates representing the shape of the lactation curve were characterized using geometric morphometrics. Genetic parameters of daily milk yield were estimated for the individual animal model using REML. Results of selection were simulated using the multivariate breeders' equation. We defined 6 selection regimens: (1) decrease in the third month in milk (MIM), (2) increase in the ninth MIM, (3) decrease in the third MIM and increase in the ninth MIM, (4) decrease in the first MIM, (5) decrease in the second MIM, and (6) decrease in the fourth MIM. The first 3 selection criteria targeted changes in the MIM of previous measurements of persistency in the Israeli Holstein population, and the last 3 targeted the months with the highest variability. All selection regimens attempted to modify the shape of the lactation curve while keeping total milk yield constant. The first 3 selection regimens showed greater total responses, with the highest response for decrease in the third MIM and increase in the ninth MIM. Magnitudes of correlated responses consistently surpassed direct responses in all selection criteria, suggesting that selection on specific parts of the lactation curve would produce correlated responses in other parts. Predicted responses to selections were depicted as shape changes from the mean shape in the data set. Most predicted responses demonstrated a fairly similar shape compared with the lactation curve. The predicted responses of lactation shapes of the last 3 selection regimens were

\footnotetext{
Received January 17, 2019.

Accepted April 4, 2019.

* Current address: Unidad de Investigación, Universidad Gerardo Barrios, San Miguel CP 3301, El Salvador.

†Corresponding author: joel.weller@mail.huji.ac.il
}

overall lower in the first 3 MIM and decreased less in the last MIM, demonstrating a much flatter shape and thus improved persistency.

Key words: geometric morphometrics, shape response, lactation curve, persistency

\section{Short Communication}

Predicting the response to selection for a given trait is essential in animal breeding and genetics. Particularly, the persistency of the lactation curve, the rate of decline after peak milk yield (Togashi and Lin, 2003; Cole and Vanraden, 2006), is an important trait with respect to farmers' profit and cows' welfare. Persistency is a property of the shape of the lactation curve, and shape is a multidimensional character. Weller et al. (2006) estimated persistency in the Israeli Holstein population as the ratio of milk yield at 270 DIM to milk yield at 90 DIM for first-parity cows. Although different measurements of persistency have been proposed [for reviews, see Gengler (1996) and Jensen (2001)], no method can simulate the response of shape as a whole and as an independent character.

Previous multivariate measurements of persistency were principal component (PC) analysis (van der Werf et al., 1998; Olori et al., 1999; Druet et al., 2005) and factor analysis (Macciotta et al., 2004, 2006) based on monthly test day records. These studies computed the eigenvectors and eigenvalues from either the additive genetic or environmental covariance matrices, and they were interpreted as measures of production level (PC1) and persistency (PC2). Togashi and Lin (2006, 2007) proposed an algorithm for genetic selection based on the eigenvectors. None of these studies considered daily production records even though these data are now available at least for milk production in many advanced production systems.

The response of shape to selection depends on the additive genetic $(\mathbf{G})$ and phenotypic $(\mathbf{P})$ covariance matrices among the different parts of the lactation curve (Klingenberg and Leamy, 2001). Durón-Benítez et al. $(2018 \mathrm{a}, \mathrm{b})$ demonstrated the combination of geo- 
metric morphometric and multivariate quantitative genetic methods for the genetic evaluation of shape of the lactation curve as a whole and as a separate character, in which the parts of the lactation shape with the maximal response to selection were identified from the GP matrix (Figure 3 in Durón-Benítez et al., 2018a). Using the GP matrix, the response to selection of shape can be predicted with the multivariate breeders' equation as implemented by Klingenberg and Leamy (2001). The objective of this study was to simulate the response to selection by selection for the shape of the lactation curve of first-parity Israeli Holstein cattle. We hypothesized that applying selection regimens in different months of production may produce different shapes for the lactation curve. The predicted response to selection of the lactation curve shape was visualized as shape changes from the mean shape in the data set.

The $\mathbf{G}$ and $\mathbf{P}$ covariance matrices used to examine the predicted response to selection on shape were obtained from the analysis of Israeli Holstein cattle (Durón-Benítez et al., 2018a). The $\mathbf{G}$ and $\mathbf{P}$ matrices correspond to the shape of the lactation curve as depicted by orbital graphs. In the geometric morphometric literature, shape refers to all the geometrical information that remains after removing information on location, size, and orientation (Kendall, 1977). Shape variables (Procrustes coordinates) were obtained from the geometric morphometric analysis on 2-dimensional landmark and semi-landmark coordinates representing lactation curves depicted by orbital graphs. Then, those shape variables were analyzed by the multitrait animal model using the MTC REML program (Misztal, 1994) to estimate the genetic parameters. Daily lactation records of 3,492 first-parity cows were analyzed. Details of data edits and corrections for environmental effects were described by Weller and Ezra (2004, 2016).

To predict the response to selection on shape, we followed the procedure developed by Klingenberg and Leamy (2001) and Klingenberg et al. (2010). They used the multivariate version of the breeders' equation $\Delta \mu$ $=\mathbf{G P}^{-1} \mathbf{s}$ (Lande, 1979), where $\boldsymbol{\Delta} \boldsymbol{\mu}$ denoted the response to selection (the difference between the mean shapes of the parental and offspring generations), $\mathbf{s}$ was a vector of selection differentials (the difference of the averages in the parental generation before and after selection), and the other terms were as defined previously. This equation was applied to the estimates of $\mathbf{G}$ and $\mathbf{P}$ covariance matrices of shape of the lactation curve (Durón-Benítez et al., 2018a).

The selection differential (s) is a vector in shape space that can be visualized and directly compared with selection response (Klingenberg and Leamy, 2001). However, the selection differentials have the disadvantage of not separating direct selection from indirect selection due to phenotypic covariation among shape variables (Klingenberg et al., 2010). Thus, the graphical display of selection differentials corresponds to total selection.

We defined 6 selection differential regimens. The first 3 were designed to simulate changes in milk production at the DIM used to compute persistency by Weller et al. (2006): (1) decrease in the third month in milk (MIM), (2) increase in the ninth MIM, and (3) decrease in the third MIM and increase in the ninth MIM. The third MIM (or landmark) corresponded to peak milk yield of first-parity cows, and the ninth MIM was 180 DIM after peak production; the third selection criterion corresponds to previous selection for persistency implemented in the Israeli selection index (Weller et al., 2006). The other 3 selection differentials were also designed to improve persistency: (4) decrease in the first MIM, (5) decrease in the second MIM, and (6) decrease in the fourth MIM. To predict the response to selection, it is necessary to first identify the aspect of the shape with the greatest response to selection (Klingenberg and Leamy, 2001); for lactation curve shape, the last 3 MIM were previously identified with the maximal response to selection from the GP matrix (Figure 3 in Durón-Benítez et al., 2018a). The magnitude for each selection differential was chosen arbitrarily as a length of 0.02 Procrustes units; this shape change is within the values of variation observed in the sample, and the resulting response is visible without amplification. All 6 selection regimens corresponded to change the shape of the lactation curve with total milk yield held constant. The selection differentials were simulated as localized shape changes - that is, changes in a single or combined monthly yield of the lactation curve.

For each of the 6 examples, the vector of total response to selection $(\boldsymbol{\Delta} \boldsymbol{\mu})$ was separated into 2 components: direct and correlated responses. The former corresponded to the direction of the shape feature under the selection gradient, and the latter corresponded to the change in the remaining shape features that were not under direct selection (Klingenberg and Leamy, 2001). The magnitude of the direct and correlated responses was quantified in units of Procrustes distance. In all other details, the analyses for the 6 selection differentials were conducted as described by Klingenberg and Leamy (2001) and computed as implemented in MorphoJ software (Klingenberg, 2011).

The magnitude of the predicted responses for the 6 selection regimens is presented in Table 1. Magnitude of predicted total responses for all selection criteria was much greater than the selection differential of magnitude 0.02 units of Procrustes distance; however, direct responses were lower than correlated responses. 
Table 1. Magnitude of predicted responses for 6 selections regimens (length unit of Procrustes distance) ${ }^{1}$

\begin{tabular}{lccc}
\hline Selection regimen $^{2}$ & $\begin{array}{c}\text { Direct } \\
\text { response }\end{array}$ & $\begin{array}{c}\text { Correlated } \\
\text { response }\end{array}$ & $\begin{array}{c}\text { Total } \\
\text { response }\end{array}$ \\
\hline Decrease in third MIM & -0.021 & 0.085 & 0.088 \\
Increase in ninth MIM & 0.00051 & 0.051 & 0.051 \\
Decrease in third and increase in ninth MIM & -0.098 & 0.097 & 0.098 \\
Decrease in first MIM & 0.027 & 0.048 & 0.055 \\
Decrease in second MIM & -0.0062 & 0.022 & 0.023 \\
Decrease in fourth MIM & 0.0020 & 0.025 & 0.025 \\
\hline
\end{tabular}

${ }^{1}$ Magnitude of selection intensity was 0.02 in all regimens.

${ }^{2} \mathrm{MIM}=$ month in milk.

Among the first 3 selection regimens, the magnitude of total predicted response was the highest (0.098) for decrease in third MIM and increase in ninth MIM; the second highest (0.088) was decrease in third MIM, and the lowest (0.051) was increase in ninth MIM. The magnitudes of the predicted responses for the other 3 selection criteria were lower. Of these, the decrease in first MIM (0.055) produced the highest total response to selection, followed by decrease in fourth $(0.025)$ and second (0.023) MIM.

The results of the predicted responses to selection depicted as shape changes from the mean shape in the data set are shown in Figure 1 for the first 3 regimens and in Figure 2 for the last 3 regimens. The first column of outlines (lactation shapes) represents the 3 selection differentials related to the measurement of persistency in the Israeli Holstein population, and the second column of outlines shows the result of simulated responses to selection. The total responses in the first 3 selection regimens included bigger changes in other regions of the lactation shape than in the selected month (black outline with solid-circle markers in the second column in Figure 1); changes were actually opposite with respect to the first and third selection differentials for third MIM. The overall patterns of variations of the responses to selections were very similar among the 3 selection regimens. For instance, they all showed the outward shift from the first to third months and the inward move in the sixth and 10th months.

The last 3 selection differentials were also intended to simulate decreases in production in the first, second, and fourth MIM, respectively (Figure 2). These MIM comprised the part of the shape identified with the highest response to selection by the first PC of the GM matrix (Durón-Benítez et al., 2018a), and they corresponded to the ascending phase of the lactation curve. Compared with the previous 3 patterns of responses to selection, the latter 3 were lower overall in the first 3 MIM and decreased less in the last MIM, demonstrating a much flatter shape and thus improved persistency. Selection differential D produced a response opposite to all the others (e.g., the decrease from first to third MIM and increase in sixth and 10th MIM). These results support the hypothesis that applying selection regimens in different months of lactation produced different responses in the shape of the lactation curve.

The localized shape changes used as selection differentials aimed to simulate selection for improving persistency by modifying shape and keeping constant the total production of the lactation curve. We obtained consistently greater correlated responses than the direct responses for all selection differentials, and the correlated responses constituted most of the total responses. This suggests that selection on localized parts of the shape of the lactation curve will produce correlated responses in other parts. The parts of the shape with greater responses coincide with the parts (beginning and end of lactation) presenting greater variation, as reported by Druet et al. (2005).

The pattern of variation reflected in the first 3 responses to selection shows increases in the ascending phase. In contrast, the pattern of variation of the other 3 responses to selection reflects a decrease in the ascending phase. Although the magnitudes of the total responses were greater overall for the first 3 regimens, the last 3 regimens were more effective at producing a flatter shape, and among the 3, targeting a decrease in the first MIM results in the flattest shape (selection differential D of Figure 2).

The combination of the 2 methods implemented in this study allowed the simulation of the response to selection for specific shape changes targeted to improve persistency of the lactation curve in Israeli first-parity Holstein cattle. Correlated responses were greater than direct responses for the 6 selection criteria. The criterion "decrease in the third MIM and increase in the ninth MIM" showed the highest magnitude of the predicted response to selection, and the predicted shape was a scaled version of the mean shape in the data set. The selection criterion "decrease in the first MIM" was effective in producing a flatter shape of the lactation curve. 

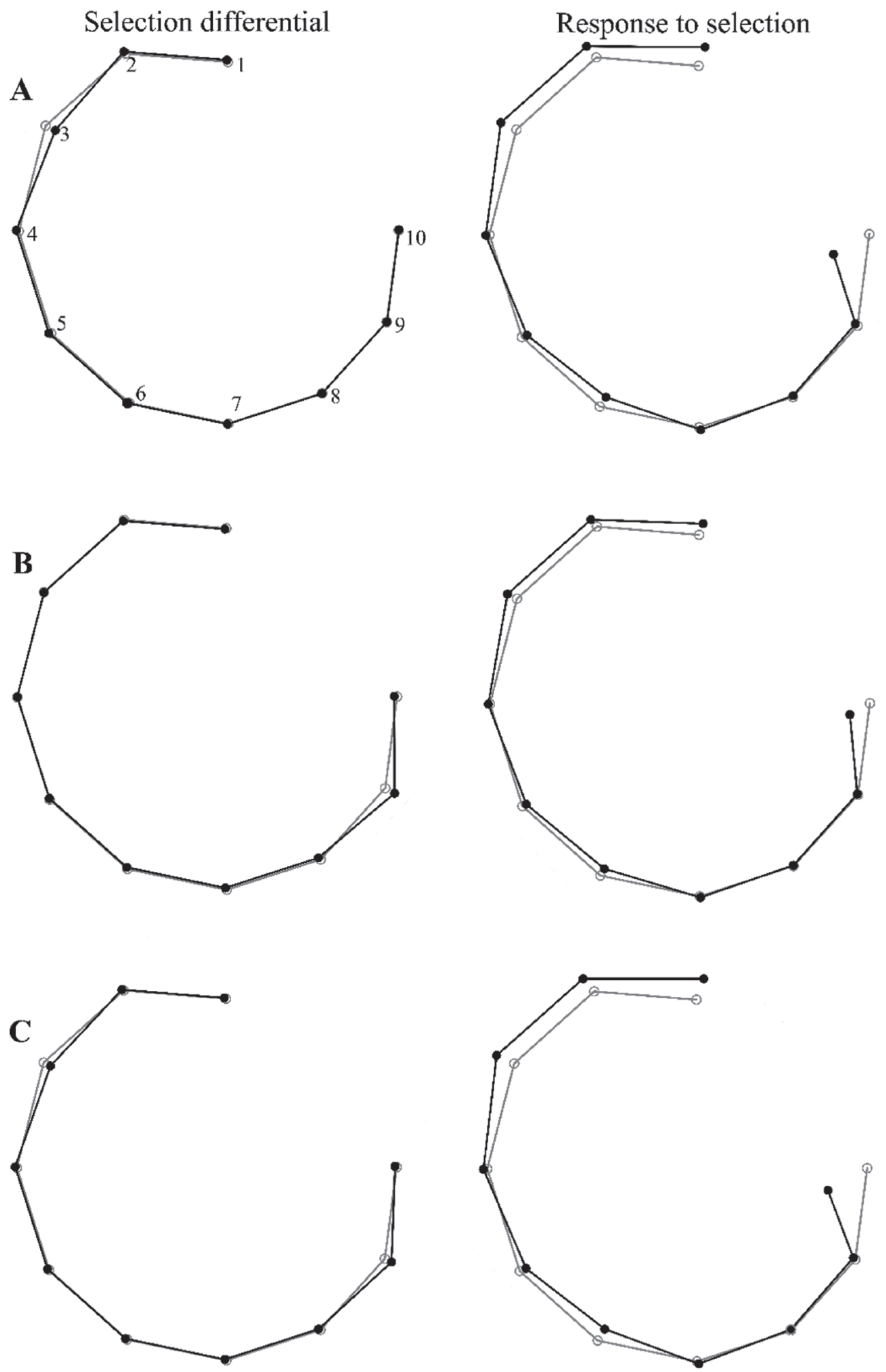

Figure 1. Response to selection of 3 differential selections on the shape of the lactation curve: (A) decrease in the third month in milk (MIM); (B) increase in the ninth MIM; and (C) decrease in the third MIM and increase in the ninth MIM. In the first column, the gray outlines with empty-circle markers show the mean lactation shape in the study, and the black outlines with solid-circle markers show the mean shape with the shifts in localized landmarks (the selection differentials drawn as shape change with a distance of 0.02 unit of Procrustes). In the second column, the gray outlines correspond to the mean lactation shape before selection, and the black outlines correspond to the mean lactation shape change after selection (scaled to 0.1 Procrustes unit). 


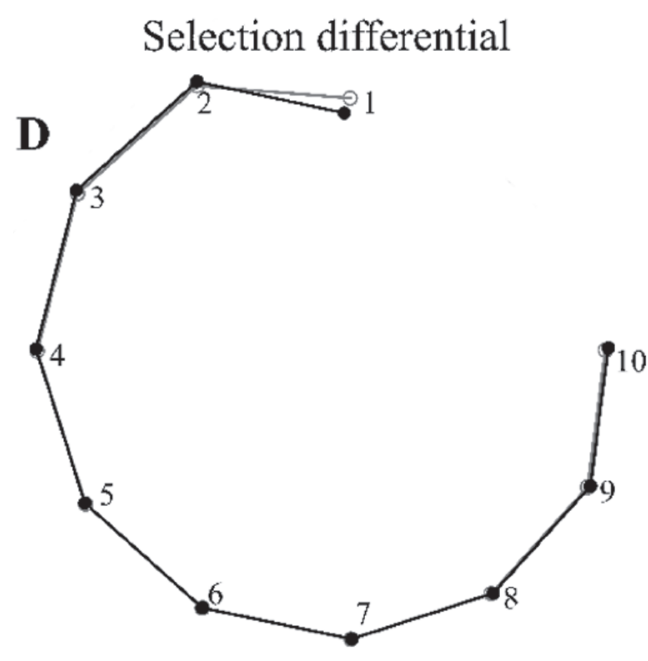

\section{Response to selection}
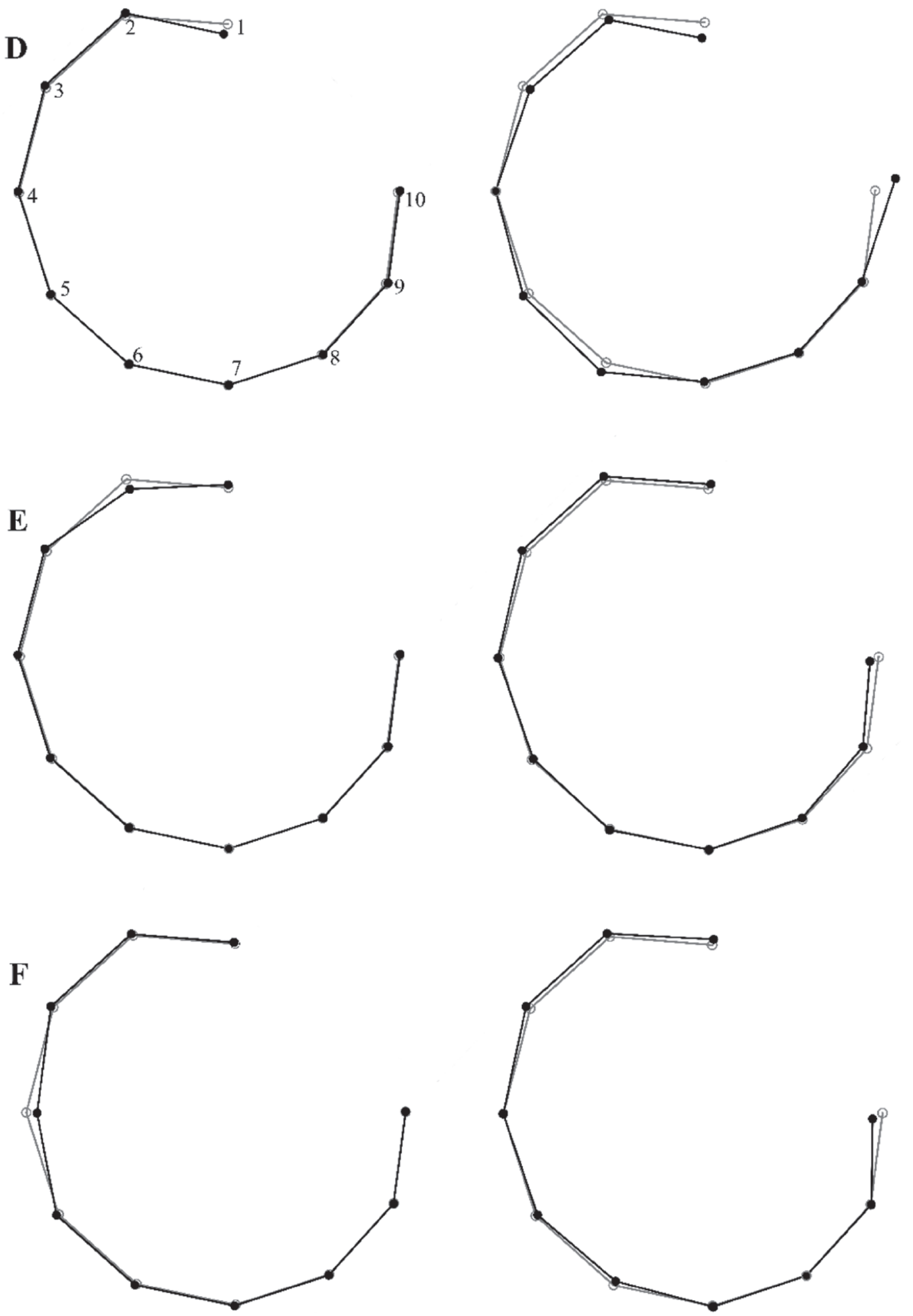

Figure 2. Response to selection of 3 differential selections on the shape of the lactation curve: (D) decrease in the first month in milk (MIM); (E) decrease in the second MIM; and (F) decrease in the fourth MIM. In the first column, the gray outlines with empty-circle markers show the mean lactation shape in the study, and the black outlines with solid-circle markers show the mean shape with the shifts in localized landmarks (the selection differentials drawn as shape change with a distance of 0.02 unit of Procrustes). In the second column, the gray outlines correspond to the mean lactation shape before selection, and the black outlines correspond to the mean lactation shape change after selection (scaled to 0.1 Procrustes unit). 


\section{ACKNOWLEDGMENTS}

This research was supported by grant number 588042-5-063F from the US-Israel Binational Agricultural Research and Development (BARD) Fund (Rishon LeZion, Israel) and by a grant from the Israel Dairy Board (Yehud, Israel). We thank Ignacy Misztal for use of the Multitrait REML Estimation of Variance Components Program by Canonical Transformation (University of Georgia, Athens) program, and the reviewers for many useful comments.

\section{REFERENCES}

Cole, J. B., and P. M. Vanraden. 2006. Genetic evaluation and best prediction of lactation persistency. J. Dairy Sci. 89:2722-2728.

Druet, T., F. Jafrezic, and V. Ducrocq. 2005. Estimation of genetic parameters for test day records of dairy traits in the first three lactations. Genet. Sel. Evol. 37:257-271. https://doi.org/10.1051/ gse:2005001.

Durón-Benítez, Á. A., J. I. Weller, and E. Ezra. 2018a. Using geometric morphometrics for the genetics analysis of shape and size of lactation curves in Israeli first-parity Holstein cattle. J. Dairy Sci. 101:11132-11142. https://doi.org/10.3168/jds.2018-15209.

Durón-Benítez, Á. A., J. I. Weller, and E. Ezra. 2018b. Heritability and genetic correlations of shape and size of lactation curves in Israeli Holsteins using geometric morphometrics. J Dairy Sci. 101(Suppl. 2):395.

Gengler, N. 1996. Persistency of lactation yield: A review. Interbull Bull. 12:87-96.

Jensen, J. 2001. Genetic evaluation of dairy cattle using test-day models. J. Dairy Sci. 84:2803-2812. https://doi.org/10.3168/jds.S0022 $-0302(01) 74736-4$.

Kendall, D. G. 1977. The diffusion of shape. Adv. Appl. Probab. 9:428-430. https://doi.org/10.1021/nn1000996.

Klingenberg, C. P. 2011. MorphoJ: An integrated software package for geometric morphometrics. Mol. Ecol. Resour. 11:353-357. https:// doi.org/10.1111/j.1755-0998.2010.02924.x.

Klingenberg, C. P., V. Debat, and D. A. Roff. 2010. Quantitative genetics of shape in cricket wings: Developmental integration in a functional structure. Evolution 64:2935-2951. https://doi.org/10 $.1111 / \mathrm{j} .1558-5646.2010 .01030 . x$.

Klingenberg, C. P., and L. J. Leamy. 2001. Quantitative genetics of geometric shape in the mouse mandible. Evolution 55:2342-2352. https://doi.org/10.1111/j.0014-3820.2001.tb00747.x.
Lande, R. 1979. Quantitative genetic analysis of multivariate evolution, applied to brain: Body size allometry. Evolution 33:402-416.

Macciotta, N. P., D. Vicario, and A. Cappio-Borlino. 2006. Use of multivariate analysis to extract latent variables related to level of production and lactation persistency in dairy cattle. J. Dairy Sci. 89:3188-3194. https://doi.org/10.3168/jds.S0022-0302(06)72593 $-0$.

Macciotta, N. P., D. Vicario, C. Di Mauro, and A. Cappio-Borlino. 2004. A multivariate approach to modeling shapes of individual lactation curves in cattle. J. Dairy Sci. 87:1092-1098. https://doi .org/10.3168/jds.S0022-0302(04)73255-5.

Misztal, I. 1994. MTC-A multitrait REML program that uses the canonical transformation but allows for several random effects. Accessed Feb. 1, 2017. http://nce.ads.uga.edu/ ignacy/oldprograms .html.

Olori, V. E., W. G. Hill, B. J. Mcguirk, and S. Brotherstone. 1999. Estimating variance components for test day milk records by restricted maximum likelihood with a random regression animal model. Livest. Prod. Sci. 61:53-63.

Togashi, K. and C. Y. Lin. 2003. Modifying the lactation curve to improve lactation milk and persistency. J. Dairy Sci. 86:1487-1493. https://doi.org/10.3168/jds.S0022-0302(03)73734-5.

Togashi, K., and C. Y. Lin. 2006. Selection for milk production and persistency using eigenvectors of the random regression coefficient matrix. J. Dairy Sci. 89:4866-4873. https://doi.org/10.3168/jds .S0022-0302(06)72535-8.

Togashi, K., and C. Y. Lin. 2007. Genetic modification of the lactation curve by bending the eigenvectors of the additive genetic random regression coefficient matrix. J. Dairy Sci. 90:5753-5758. https:// doi.org/10.3168/jds.2007-0363.

van der Werf, J. H. J., M. E. Goddard, and K. Meyer. 1998. The use of covariance functions and random regressions for genetic evaluation of milk production based on test day records. J. Dairy Sci. 81:3300-3308. https://doi.org/10.3168/jds.S0022-0302(98)75895 $-3$.

Weller, J. I., and E. Ezra. 2004. Genetic analysis of the Israeli Holstein dairy cattle population for production and nonproduction traits with a multitrait animal model. J. Dairy Sci. 87:1519-1527. https: //doi.org/10.3168/jds.S0022-0302(04)73303-2.

Weller, J. I., and E. Ezra. 2016. Genetic and phenotypic analysis of daily Israeli Holstein milk, fat, and protein production as determined by a real-time milk analyzer. J. Dairy Sci. 99:9782-9795. https://doi.org/10.3168/jds.2016-11155.

Weller, J. I., E. Ezra, and G. Leitner. 2006. Genetic analysis of persistency in the Israeli Holstein population by the multitrait animal model. J. Dairy Sci. 89:2738-2746. https://doi.org/10.3168/jds S0022-0302(06)72350-5. 